Recherches en didactique des langues et des cultures

Les cahiers de l'Acedle

$12-3 \mid 2015$

Cultures de recherche en linguistique appliquée

\title{
Pluri-inter-transdisciplinarité des recherches impliquées dans la description de la langue en usage
}

Comment penser la porosité des champs de recherche?

Catherine Carlo

\section{OpenEdition}

\section{Journals}

Édition électronique

URL : http://journals.openedition.org/rdlc/959

DOI : $10.4000 /$ rdlc. 959

ISSN : 1958-5772

Éditeur

ACEDLE

Référence électronique

Catherine Carlo, «Pluri-inter-transdisciplinarité des recherches impliquées dans la description de la langue en usage », Recherches en didactique des langues et des cultures [En ligne], 12-3 | 2015, mis en ligne le 07 décembre 2015, consulté le 19 avril 2019. URL : http://journals.openedition.org/rdlc/959 ; DOI : $10.4000 /$ rdlc. 959

Ce document a été généré automatiquement le 19 avril 2019

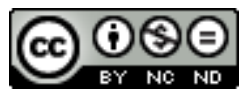

Recherches en didactique des langues et des cultures is licensed under a Creative Commons AttributionNonCommercial-NoDerivatives 4.0 International License 


\section{Pluri-inter-transdisciplinarité des recherches impliquées dans la description de la langue en usage}

Comment penser la porosité des champs de recherche?

\section{Catherine Carlo}

\section{Introduction}

1 Le rapprochement interdisciplinaire n'est pas une démarche récente. Le recours à l'emprunt de catégories, questions de recherche, perspectives, à des disciplines ou domaines connexes, est en effet une conduite heuristique aussi spontanée qu'ancienne dans l'émergence des idées (création de l'Université comme lieu de l'universalité au moyen âge, mouvement encyclopédique au 18ème etc.). La décentration du chercheur par rapport à ces objets d'investigation initiaux et paradigmes de référence joue, par ailleurs, depuis toujours, un rôle dans la construction de connaissances. Il n'en reste pas moins que cette démarche est sommée d'établir sa légitimité, tant le mode de contact et la maitrise (supposée approximative) des disciplines mises en lien par ceux qui s'y risquent, sont parfois tenus en suspicion. Défiance qui touche à la fois l'ethos du passeur de frontières lorsqu'il n'est pas un des grands nomades de la connaissance (la question ne se pose pas quand il s'agit de Pascal ou de Piaget par exemple), défiance qui touche également à son expertise, défiance enfin concernant l'efficience de la démarche.

\section{Des notions en débat}

2 La question du bien-fondé épistémologique et celle de l'intérêt praxéologique de la collaboration des disciplines et des champs de recherche ont été au cœur des débats en sciences humaines, au milieu des années 70. Ces questions ont été réactivées par la suite, notamment par les travaux d'Edgar Morin (1982) portant sur la pensée complexe qui cherche à se donner les moyens conceptuels de rendre intelligible la complexité du réel. 
Elles sont traversées de controverses et sont, en conséquence, marquées par des différends terminologiques concernant la définition et le périmètre des notions de pluridisciplinarité ou multidisciplinarité, interdisciplinarité et transdisciplinarité : des termes récurrents sous la plume de différents auteurs. Au-delà des distinctions qui sont parfois grandes (voir la recension de Creutzer, 2002), se dessine l'idée générale d'une gradation des types de collaboration entre les disciplines, qui va de la simple juxtaposition de travaux issus de disciplines ou de cadres de référence différents dans la même discipline et gardant leur autonomie (pluri et multidisciplinarité), jusqu'à une dilution radicale des frontières, des objets, des concepts, des méthodes des disciplines, sous l'effet de leur recomposition réciproque (ce qui est généralement entendu par le terme de transdisciplinarité). La voie moyenne est celle de l'interdisciplinarité, qui établit des connexions plus ou moins denses, au plan méthodologique ou conceptuel entre les disciplines.

Dans le domaine qui est le nôtre, la didactique des langues étrangères, qui côtoie d'autres sous-disciplines des sciences du langage, mais aussi différentes disciplines relevant des sciences humaines, on peut se demander quelle actualité revêt cette question de l'interdisciplinarité, avec l'émergence de disciplines contributives nouvellement considérées, avec les perspectives ouvertes par la technologie et avec une évolution, voire des injonctions nouvelles de la politique de la recherche. De la pluridisciplinarité (ou multidisciplinarité, sans distinguo), qui consiste en une juxtaposition des travaux de disciplines différentes, sans interactions entre elles, il y a peu à dire, si ce n'est, comme le souligne Charaudeau (2010), que ce premier stade de rencontre constitue déjà une « entreprise salutaire».

4 À l'autre bout de la chaîne, le niveau le plus élevé de collaboration, la transdisciplinarité, qui aboutirait à la construction d'un espace de recherche holistique, qu'on l'envisage dans l'acception de Piaget (1972) à qui on attribue généralement la paternité de la notion ou dans l'acception de Morin (1994), constitue un horizon de recherche à venir, une réflexion programmatique, plutôt qu'une démarche effectivement mise en œuvre permettant de subsumer dans un même ensemble, des recherches sectorielles.

5 Rappelons que pour Piaget la transdisciplinarité prend la forme d'une théorie générale des systèmes :

(...) enfin, à l'étape des relations interdisciplinaires, on peut espérer voir se succéder une étape supérieure qui serait "transdisciplinaire», qui ne se contenterait pas d'atteindre des interactions ou réciprocités entre recherches spécialisées mais situerait ses liaisons à l'intérieur d'un système total sans frontières entre disciplines $(1972: 170)$.

La transdisciplinarité selon E. Morin, qui revêt une grande ambition, est posée, fort lyriquement, comme une posture de recherche à conquérir. Traversant les cloisonnements disciplinaires et s'appuyant sur des concepts transversaux (opérateurs de reliance), elle est présentée comme une condition de la compréhension globale du réel (et c'est sa raison d'être) : "La transdisciplinarité se caractérise souvent par des schèmes cognitifs traversant les disciplines, parfois avec une violence telle qu'ils les mettent en transe » $(1994: 8)$. Nous posons pour notre part que c'est l'état de maturité des disciplines (qui nécessite donc un état des lieux) qui conduit à envisager leur degré potentiel de rapprochement sur un objet complexe ou projet commun qu'elles se donnent. Compte tenu de l'état de développement des disciplines qui nous mobilisent en sciences humaines, c'est, de fait, sur la question de l'interdisciplinarité que semble devoir se focaliser la réflexion. 


\section{Une interdisciplinarité «focalisée »}

6 Dans un article de synthèse de 2010, Charaudeau qui reprend à son compte la distinction générale entre pluridisciplinarité, interdisciplinarité et transdisciplinarité, qu'on vient de mentionner, s'attache particulièrement à donner un contenu à la notion d'interdisciplinarité en sciences humaines, en mettant l'accent sur sa dimension épistémologique. Il rappelle d'abord qu'il ne saurait y avoir d'interdisciplinarité, sans identification initiale, pour les disciplines ou sous-disciplines sollicitées :

- des présupposés théoriques sur lesquels elles se fondent ;

- des méthodologies qu'elles utilisent ;

- des modes d'interprétations des résultats recueillis qu'elles privilégient.

7 C'est en effet à partir de ses propres fondements, outillage méthodologique, et cadre interprétatif que chaque discipline ou sous-discipline appréhende notions communes, problématisations et analyse des résultats. C'est en conséquence en tenant compte des cadres théoriques identifiés, pour éviter leur "gauchissement", que peuvent être questionnés, et éventuellement empruntés, notions, concepts et outils d'analyse. Dit autrement, il ne peut y avoir d'interdisciplinarité que s'il existe des disciplines constituées (Annick Louis, 2013).

Charaudeau montre, ensuite, à titre d'exemple, comment la sociologie, la psychologie sociale, les sciences du discours et de la communication, qui s'attachent toutes à l'analyse de phénomènes sociaux, peuvent partager :

- d'une part, des notions communes : identité (notion qui marque le retour du sujet sur la scène de la recherche en sciences humaines), stratégie, influence, représentations, imaginaires sociaux), sans qu'il y ait de «prés carrés » légitimes ;

- d'autre part, des cadres de questionnement (problématisation des processus de régulation sociale, problématisation des rapports de force, problématisation de la construction des savoirs) ;

- mais aussi, des outils d'analyse transversaux. Et Charaudeau n'entend pas seulement par là des techniques informatiques ou statistiques qui permettent désormais de traiter des données massives et de ce fait, transforment les études quantitatives et modifient la constitution des corpus. Il considère plus fondamentalement deux démarches méthodologiques, éventuellement complémentaires: une démarche empirique, qui construit son objet à partir de l'observation de phénomènes sociaux, sur la base d'un recueil de données et une démarche hypothético-déductive, qui cherche à valider, sur un terrain donné, des hypothèses posées et les catégories interprétatives ;

- enfin, des protocoles d'interprétation des résultats, axés sur une recherche interne des chaînes causales, ou sur l'interprétation externe qui confronte des résultats à ceux de disciplines ayant le même objet.

9 En bref: "Chaque discipline opère une ponction dans chacun des domaines (les phénomènes sociaux) créant ainsi un lieu de pertinence d'analyse qui lui est propre. La question demeure cependant celle de leur articulation possible» $(2010: 6)$. Pour penser cette articulation des disciplines, Charaudeau propose la notion d'interdisciplinarité «focalisée », qui identifie, au cas par cas, le niveau de rapprochement visé (niveau des notions/concepts, outils, résultats). Il précise que ce type d'interdisciplinarité ne peut être mis en œuvre sans risque de confusion, qu'entre deux disciplines, quitte à réitérer la mise en regard de disciplines, deux à deux. 
10 Je reprends à mon compte cette approche de l'interdisciplinarité, à condition d'ajouter qu'elle ne saurait se mettre en place sans la recension de phénomènes déviants, rétifs à entrer dans les cadres explicatifs disponibles et sans la promotion d'un modèle paradoxal de chercheur, que je propose de qualifier de généraliste en un sens qu'il faut maintenant préciser.

\section{Le chercheur généraliste, un oxymore?}

11 "La spécialisation du travail s'accompagne d'un processus de "professionnalisation" qui constitue sans contexte un gain de rigueur et de précision scientifique. On a donc fini par associer " spécialisation et scientificité" ", dit Lahire (2012 : 342). En effet, pour tenter de produire des données et des connaissances nouvelles, une maitrise précise spécialisée d'un champ de recherche est une condition sine qua non mais cet état de fait s'accompagne d'un paradoxe : cette maitrise ne suffit pas à entrer dans une démarche interdisciplinaire, fûtelle «focalisée ».

Pour ce faire, il est nécessaire d'appréhender le cadre de référence d'autres disciplines, à un niveau de compétence identifié qui garantisse l'intercompréhension disciplinaire. Il s'agit, autrement dit, de s'engager dans une démarche d'apprentissage qui n'est pas dans le droit-fil d'une spécialisation. Ceci n'entraine nullement le retour à un modèle de chercheur au discours globalisant, qui exercerait une grille d'interprétation unique sur des phénomènes à travers les disciplines et sous-disciplines qu'il approche mais ceci implique d'adopter une posture d'apprentissage d'une autre ou d'autres disciplines ou sous-disciplines, avec la perspective de "créer des ponts» et de remanier ses catégorisations. On qualifiera cette posture de "généraliste» dans la mesure où elle inscrit les connaissances disciplinaires, parcellaires, dans un projet plus vaste d'appréhension du réel et de flexibilité des catégorisations. Vaste tâche ? Rien n'est moins sûr. De même qu'un plurilingue ne maîtrise pas au même degré les langues qu'il parle, le chercheur généraliste n'est pas tenu, pour autant, de s'approprier le cadre de référence d'autres disciplines ou sous-disciplines avec le degré de maîtrise qui est le sien dans sa discipline d'origine.

Pour des raisons institutionnelles, dans le domaine de recherche qui est le nôtre, le double statut d'enseignant et de chercheur a ceci de particulier qu'il a des incidences sur le périmètre de recherche investi et qu'il favorise, de fait, une approche interdisciplinaire. Pour donner un exemple, tel enseignant-chercheur spécialiste de l'acquisition des langues, dont la recherche initiale porte sur les mécanismes cognitifs et les conduites communicatives des apprenants en langue étrangère, est conduit, en quelque sorte par contiguïté, à s'intéresser aux recherches de nature didactique, et à se pencher, après s'être intéressé à l'apprenant, sur ce qui se passe du côté des pratiques d'étayage mises en œuvre par l'enseignant et les pairs.

Ces considérations conduisent à insister sur le caractère difficilement "décrété » de la démarche interdisciplinaire, qui suppose un temps d'appropriation de connaissances et par voie de conséquence, à marteler la nécessité de l'intégration de cette contrainte temporelle dans une politique de la recherche qui ne peut se contenter de promouvoir l'interdisciplinarité de façon incantatoire. 


\section{De la pluridisciplinarité à l'interdisciplinarité focalisée}

15 les deux cas, une démarche évolutive. Dans le premier cas, comme dans le second, le
degré de collaboration ne peut se déterminer a priori et résulte d'un état des lieux des ressources des disciplines en présence.

17 Le premier exemple concerne un projet qui se met en place autour du type discursif « cours magistral à l'Université ». La demande sociale concernant la formation en F.O.U. (acronyme malencontreux qui désigne le Français sur Objectif Universitaire) est forte mais, en amont, se pose au plan de la recherche qui nous concerne ici, la question de la description d'un type de discours.

La raison du rapprochement des disciplines sur cet objet, au travers des chercheurs qui ont convenu de l'aborder (projet Idali 2013) est le double constat d'un changement contextuel et social de l'Université et d'un possible renouvellement du genre discursif, qui n'a pas été revisité de façon globale d'un point de vue descriptif depuis l'étude d'Ali Boucha (1984). D'une part, en effet, les universités périphériques accueillent des publics maîtrisant sans doute moins que les générations précédentes, par imprégnation, les codes du type magistral auquel ils sont confrontés; d'autre part, compte tenu de la prise en compte de ces « nouveaux publics » et de l'arrivée des Technologies de l'Information et de la Communication pour l'Enseignement (Tice), il y a tout lieu de penser que les traits marquants du type discursif «cours magistral» actuellement pratiqué doivent faire l'objet d'une description fine et actualisée. Dans une phase exploratoire, il a été envisagé une collaboration a minima sur un objet ressortissant à plusieurs disciplines et présentant un intérêt praxéologique manifeste. La juxtaposition des points de vue diversifiés, sorte d'accumulation initiale, en faisant cohabiter plusieurs perspectives spécifiques sur un même objet complexe permet une appréhension sinon globale, du moins plus large, d'un objet qui ne ressortit pas à une seule discipline. Elle expose chacun des points de vue - qui en tireront partie ou non - à des concepts, méthodes d'analyse, modes d'explication et d'interprétation qui peuvent avoir, pour chacun d'eux, une valeur heuristique. Ce pari initial, qui relève classiquement de la pluridisciplinarité, s'ancre dans la constitution d'un corpus commun. Le corpus initial réuni constitué de cours magistraux en sciences humaines (communication, psychologie cognitive, économie) doit être élargi à des cours magistraux en sciences «dures » et mis en ligne, à l'instar de ce qui a été fait pour l'anglais académique (p. ex : projet Micase de l'université de Michigan). Compte tenu des perspectives de l'équipe pluridisciplinaire dont chacun des membres est bi-disciplinaire, l'analyse des cours magistraux dispensés dans des établissements supérieurs sera abordée en se focalisant sur plusieurs entrées relevant d'une part de la recherche sur l'acquisition des langues (l'appropriation discursive dans une forme spécifique d'interactivité, les conduites d'étayage), d'autre part, de l'analyse du discours (la question des supports et de la multimodalité, de l'hétérogénéité discursive des pratiques discursives) et enfin, de la didactique des langues (l'agir professoral de l'enseignant, les cultures éducatives et discursives selon les disciplines). À terme donc, la question qui se pose est celle du passage de la pluridisciplinarité à l'interdisciplinarité focalisée, qui peut naître de la confrontation des théorisations des différents cadres de référence dans la même 
discipline ou dans des disciplines connexes. Cette interdisciplinarité, dont, en l'espèce, on ne préjuge pas de l'existence, pourrait être «focalisée » sur plusieurs objets (concepts, outils, cadre interprétatifs). L'interdisciplinarité, qui suppose des remaniements conceptuels ciblés et/ou des emprunts méthodologiques raisonnés pour chacune des disciplines considérées, ne peut advenir qu'à un stade de maturation de la réflexion commune.

Le second exemple, emprunté à Oger et Ollivier-Yaniv (2007) concerne la construction d'une démarche commune entre analyse du discours $(\mathrm{AD})$ et sociologie compréhensive qui s'appuie sur un point de convergence : la place du discours dans les deux traditions théoriques. La sociologie avec le "retour du sujet», connaît depuis une trentaine d'années « un tournant existentiel» (Touraine, 2005) qui la conduit à s'attacher aux discours produits par les sujets de l'action. De son côté l'analyse du discours s'est intéressée progressivement à des problématiques proches de l'ethno-sociologie et notamment à des "rituels langagiers dans des communautés restreintes ", en venant ainsi à " réfléchir sur l'articulation entre le sujet du discours et le sujet, tel que peuvent l'appréhender sociologues et psychologues» (Maingueneau, 1996: 11). Malgré cette proximité épistémologique, les auteurs, issus de l'AD, montrent à l'occasion d'une approche conjointe sur le discours tenu par les militaires sur leur communication institutionnelle, qu'une commune pratique de l'entretien dans les deux disciplines ne garantit pas une commune conception du sujet, de la parole, et du rapport de la parole à l'activité dans l'analyse du corpus commun de textes et entretiens. On voit là qu'il ne suffit pas de constituer un corpus commun pour qu'une interdisciplinarité se mette en place, que la compréhension spontanée des concepts issus d'autres cadres de références peut faire obstacle, conformément à l'apophtegme prêté à Culioli qui dit que «la compréhension est un cas particulier du malentendu » (1990 : 39). L'interdisciplinarité focalisée, en l'occurrence sur des concepts et catégories d'analyse, apparaît donc comme à construire.

\section{Pour conclure}

Le cheminement qui va de la pluridisciplinarité, premier engagement collectif et souvent programmatique dans une démarche de collaboration des disciplines, à l'interdisciplinarité «focalisée ", démarche de production de connaissances nouvelles à moyen terme, résulte de l'élaboration, de la construction et de l'ajustement dans l'action, au cas par cas, d'une recherche partiellement commune. Nous souscrivons à l'idée qu'il dépend d'abord de la dynamique d'appropriation par les chercheurs concernés des cadres de référence d'une autre discipline, dans la mesure où le rapprochement s'opère d'abord entre deux disciplines, quitte à se colleter, une fois cette maitrise acquise et si faire se peut, à une troisième, voire n-ième discipline. Il dépend ensuite de l'émergence de concepts peu à peu partagés ou remaniés, du réexamen potentiel des méthodes d'analyse initiales et de l'explicitation des protocoles d'interprétation des résultats. Il est rendu possible par l'adhésion, en amont, de tous les acteurs concernés au postulat ouvert d'une multi-appartenance disciplinaire de l'objet d'étude et de sa potentielle reconfiguration, conformément à une perspective constructiviste de la recherche scientifique. De ce point de vue, ce qui vaut pour la biologie, vaut pour les sciences humaines : « La nature n'est pas elle-même découpée et répartie en objet et phénomènes scientifiques. C'est la science qui constitue son objet » (Canguilhem, 1968). Enfin, qu'elle résulte d'une demande sociale externe ou d'une proximité épistémologique entre disciplines, la dynamique interdisciplinaire ainsi 
entendue en didactique des langues ne change pas de nature, dès lors qu'elle réunit les mêmes conditions de mise en œuvre (chercheurs acquis à l'idée du caractère indispensable mais néanmoins parcellaire des résultats disciplinaires et corrélativement acquis à l'idée d'une appréhension plus large du réel, durée de maturation et de réalisation suffisante de l'élaboration collective, constitution de corpus commun).

\section{BIBLIOGRAPHIE}

Ali Bouacha, M. (1984). Le discours universitaire - la rhétorique et ses pouvoirs. Berne : Peter Lang.

Berger, G. (1972). « Opinions et réalités » In Ocde (dir.). L'interdisciplinarité : problèmes

d'enseignement et de recherche dans les universités. Paris : Ocde.

Canguilhem, G. (1968). Études d'histoire et de philosophie des sciences, Paris : Vrin.

Charaudeau, P. (2010). Pour une interdisciplinarité « focalisée » dans les sciences humaines et sociales. Questions de Communication, vol 17. pp 195-222, Disponible en ligne. http:// questionsdecommunication.revues.org/385

Creutzer, M. (2002). « Aspects de l'interdisciplinarité : essai de reconstitution d'un débat ». In Gélineau, L. (dir.). L'interdisciplinarité et la recherche sociale appliquée - Réflexions sur des expériences en cours. Université de Montréal, université de Laval et Chaire d'études sur la condition des femmes. pp. 7-39. Disponible en ligne. www.fas.umontreal.ca/sha:documents/ interdisciplinarité.pdf

Culioli, A. (1990). Pour une linguistique de l'énonciation. Paris : Ophrys.

Lahire, B. (2012). Monde Pluriel - Penser l'unité des sciences sociales. Paris : Seuil.

Lahire, B. (1998). À propos de L'Homme pluriel. Interview sur le site Nathan Université. Paris : Nathan Université. Disponible en ligne. http://homme-moderne.org/societe/socio/blahire/ entrevHP.html

Louis, A. (2013). «Ce que l'enquête fait aux études littéraires : à propos de l'interdisciplinarité ». Fabula / Les colloques, Littérature et histoire en débats. Disponible en ligne. http://www.fabula.org/ colloques/document2097.php

Morin, E. (1982/1990). Science avec conscience, Paris : Fayard.

Morin, E. (1994). « Sur l'interdisciplinarité ». Bulletin Interactif du Centre International de Recherches et Études transdisciplinaires (Ciret), $\mathrm{n}^{\circ}$ 2. Disponible en ligne. http://www.iiac.cnrs.fr/ CentreEdgarMorin/spip.php?article367

Oger, C. \& Ollivier-Yaniv, C. (2007). « Analyse du discours et sociologie compréhensive. Retour critique sur une pratique de recherche transdisciplinaire ». In Bonnafous, S. \& Temmar, M. (dir.). Analyse du discours en sciences humaines et sociales. Paris : Ophrys. pp. 39-55.

Piaget, J. (1972). «L'épistémologie des relations interdisciplinaires ». In Ocde (dir.). L'interdisciplinarité - problèmes d'enseignement et de recherche dans les universités. Paris, Ocde. pp.131-141. Disponible en ligne. http://www.fondationjeanpiaget.ch/fjp/site/textes/VE/ jp72_epist_relat_interdis.pdf 


\section{RÉSUMÉS}

Le changement de paradigme qui a imposé la dénomination de sciences du langage, a abouti à faire une place, dans le champ, à des disciplines, telle la didactique des langues, jusqu'alors «sœur converse» du domaine. Cette intégration nouvelle ne dispense cependant pas de s'interroger sur les conditions et sur la réalité d'une porosité des sous-disciplines et des disciplines connexes des sciences du langage et donc sur la réalité de la pluri-inter-transdisciplinarité, ailleurs débattue (Creutzer, 2002).

De la pluridisciplinarité, qui juxtapose, sur un même objet de recherche, les points de vue de disciplines gardant leur autonomie (Charaudeau, 2010), à la transdisciplinarité qui aboutit à un espace de recherche holistique «système total sans frontières entres disciplines » (Piaget, 1972), en passant par l'interdisciplinarité qui établit des connexions plus ou moins denses au plan conceptuel et méthodologique, le spectre potentiel des partenariats entre disciplines et sousdisciplines est large.

À défaut de dresser un état des lieux de l'hybridation des sous-disciplines ressortissant aux sciences du langage et des disciplines qu'elles sont amenées à rencontrer, nous présentons quelques scenarii qui nous semblent émergents, en didactique des langues et qui tiennent à une situation spécifique des acteurs du champ, souvent spécialistes d'une des sous-disciplines des sciences du langage et, dans le même temps, didacticiens, voire enseignants de langue.

A paradigm shift imposed a new name, Language Sciences, and this led to introduce language teaching theory in the field. Nevertheless, this inclusion of new disciplines does not enable us to exempt from considering the question of pluri-inter-trans disciplinarity (Creutzer, 2002). It is necessary to question, in an epistemological view point, the porosity of sub-disciplines. We shall present some specific situations of collaboration between sub-disciplines, connected with the plurality of roles of researchers who most often are both linguists, specialists of didactics and teachers.

\section{INDEX}

Mots-clés : pluridisciplinarité, interdisciplinarité, porosité des disciplines

Keywords : pluridisciplinarity, interdisciplinarity, porosity of subdisciplines

\section{AUTEUR}

\section{CATHERINE CARLO}

Université Lumière Lyon 2, Icar UMR 5191, France.

Catherine Carlo est professeure à l'université Lumière Lyon 2. Elle était auparavant maître de conférences en sciences du langage à l'université Paris8-Vincennes-Saint-Denis, où elle a codirigé le master Didactique des langues. Membre du Diltec (Didactique des Langues, des Textes et des Cultures), EA 2288, ses travaux ont porté d'une part sur les liens entre acquisition en L2 et interactions en contexte institutionnel et d'autre part, sur les rapports entre acquisition en L2 et didactique des langues, puis se sont orientés vers l'exploration des répertoires d'enseignant et de l'agir professoral. Elle est désormais membre du laboratoire Icar, UMR 5191 (Interactions, Corpus, Apprentissages, Représentations). Courriel : catherine.carlo[at]univ-lyon2.fr 
Toile : http://icar.univ-lyon2.fr/membres/ccarlo/

Adresse : Université Lumière Lyon 2, 16 quai Claude Bernard, 69365 Lyon cedex France et Icar : ENS de Lyon-Site Descartes 15, Parvis René Descartes, BP 7000, 69342 Lyon cedex 07. 